\title{
ECOLOGIA DA PESCA ARTESANAL NO MÉDIO RIO TOCANTINS, IMPERATRIZ (MA)
}

\section{Mauricio Cetra}

Dissertação apresentada à Escola de Engenharia de São Carlos da Universidade de São Paulo, como parte dos requisitos para a obtenção do título de Mestre em Engenharia. Área de concentração: Engenharia Ambiental

ORIENTADOR: Prof. Dr. Miguel Petrere Jr.

UNESP - Depto de Ecologia

Caixa Postal 199

13506-900-Rio Claro (SP)

São Carlos

1998 
À Dedé, Raísa e Gabriel 
O homem chega e já desfaz a natureza tira gente, põe represa, diz que tudo vai mudar...

(Sá e Guarabira) 


\section{AGRADECIMENTOS}

Agradeço primeiramente a Deus, força criadora desta natureza que é fonte de meu trabalho.

Ao Prof. Dr. Miguel Petrere Jr. por mais uma orientação, ter me incentivado a continuar na vida acadêmica, ter conseguido a implantação do sistema de desembarque em Imperatriz, fornecer os dados deste trabalho, assim como ser um referencial profissional.

A Prof ${ }^{a}$. Miriam Leal de Carvalho por ter conseguido a aprovação da implantação do sistema de desembarque pesqueiro em Imperatriz e minha ida até a área de estudo.

Ao Ministério do Meio Ambiente e ao CNPq pelo apoio financeiro.

A Prof ${ }^{\mathrm{a}}$. Dr ${ }^{\mathrm{a}}$. Maria Aparecida Pion Abuabara pelas valiosas sugestões apontadas durante o exame de qualificação e o manuscrito final.

Ao Prof. Dr. Mauro César Lambert de Brito Ribeiro por ter propiciado uma ótima discussão quando da apresentação deste trabalho e pela disposição de ter se deslocado de Brasília até São Carlos para participar da banca examinadora.

Ao professor Raimundo, ao Júlio, aos outros pescadores, marreteiros e à Colônia de Pescadores Z-29 pela transmissão do conhecimento do que é a pesca no dia a dia.

Aos amigos Saul, Dadão, Benê, Carlão, Carolina, Serguei e Silvana pelas idéias trocadas que me ajudaram muito.

Ao Alberto, Elói, Dorinha e Rita por me ajudarem a enxergar novos caminhos.

Aos meus pais e irmãos que sempre me incentivaram a continuar por este caminho. 


\section{SUMÁRIO}

LISTA DE FIGURAS............................................................................... vi

LISTA DE TABELAS............................................................................................ viii

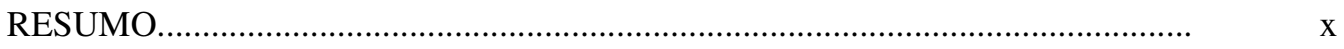

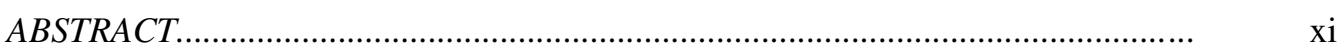

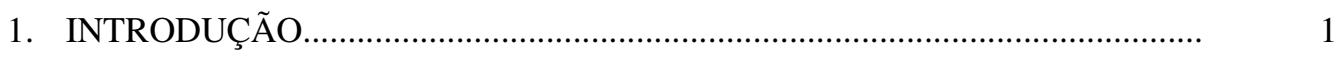

2. CARACTERIZAÇÃO GERAL DA ÁREA....................................................... 7

3. METODOLOGIA.............................................................................. 10

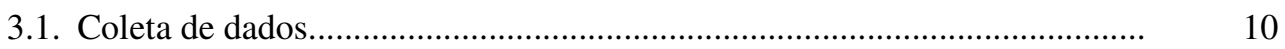

3.2. Tabulação dos dados................................................................................ 10

3.3. Composição específica das saladas de primeira e segunda............................... 11

3.4. Análise de regressão................................................................................... 12

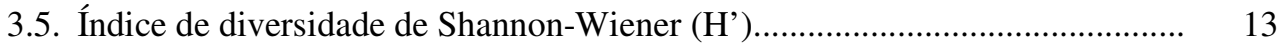

3.6. Variação mensal do desembarque.................................................................. 13

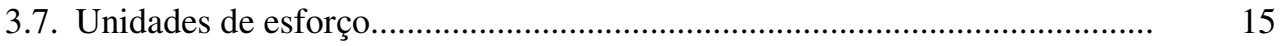

3.8. Estimativa da abundância do Prochilodus nigricans - curimatá..................... 16

3.8.1. Método de Leslie..................................................................................... 17

3.8.2. Método de De Lury.......................................................................... 20

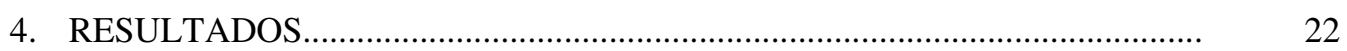

4.1. Caracterização da pesca artesanal no médio rio Tocantins.............................. 23

4.2. Análise do desembarque pesqueiro............................................................. 25

4.3. Comparação entre a captura de 1988 e 1997 na época de cheia....................... 37

4.4. Análise dos aparelhos de pesca e suas unidades de esforço............................ 40

4.5. Relações entre apetrechos de pesca e espécies mais importantes...................... 44

4.6. Análise do desembarque e estimativa da abundância do Prochilodus

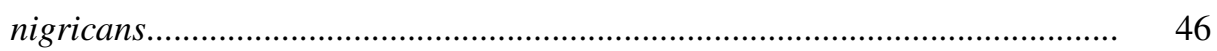

5. DISCUSSÃO ................................................................................. 50

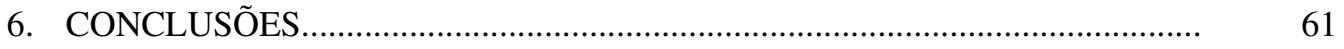

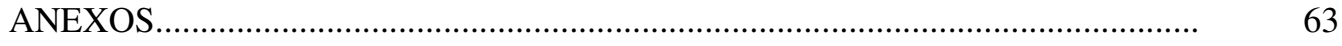

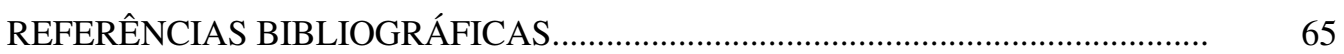




\section{LISTA DE FIGURAS}

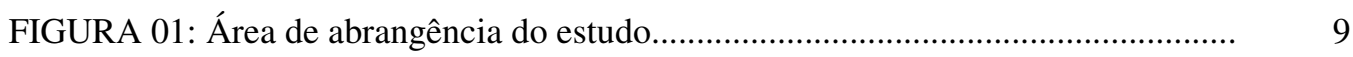

FIGURA 02: Captura ( $\mathrm{t}$ ) mensal em relação ao nível $(\mathrm{cm})$ do rio Tocantins................ 26

FIGURA 03: Nível do rio Tocantins (NIVEL $(\mathrm{cm}))$ versus captura (CAPT ( $\mathrm{t})$ ).......... 26

FIGURA 04: Esforço ( ${ }^{\circ}$ pescadores) mensal em relação ao nível $(\mathrm{cm})$ do rio Tocantins

FIGURA 05: Nível do rio Tocantins (NIVEL (cm)) versus esforço $\left(\operatorname{PESC}\left(\mathrm{n}^{\circ}\right.\right.$ de pescadores)

FIGURA 06: Captura ( $\mathrm{t}$ ) mensal em relação ao esforço ( $\mathrm{n}^{\mathrm{o}}$ de pescadores) ................. 30

FIGURA 07: Esforço (PESC ( ${ }^{\circ}$ de pescadores)) versus captura (CAPT $\left.(\mathrm{t})\right) \ldots \ldots \ldots \ldots \ldots . . . . \quad 30$

FIGURA 08: Participação relativa (\%) das famílias na composição da ictiofauna comercializada...

FIGURA 09: Número de espécies por família na ictiofauna comercializada

FIGURA 10: Relação entre a Abundância relativa (\%) e as espécies em "rank" nos meses de janeiro, março e maio de 1988

FIGURA 11: Relação entre a Abundância relativa (\%) e as espécies em "rank" nos meses de julho, setembro e dezembro de 1988 .

FIGURA 12: Nível do rio Tocantins (NIVEL $(\mathrm{cm})$ ) versus diversidade de espécies desembarcadas (H' (bits/indivíduo)).

FIGURA 13: Participação relativa (\%) das espécies comerciais mais importantes.

FIGURA 14: Participação relativa (\%) das espécies comerciais mais importantes nos meses de março, abril e novembro de 1988

FIGURA 15: Participação relativa (\%) das espécies comerciais mais importantes nos meses de março, abril e novembro de 1997.

FIGURA 16: Diagrama de Euler-Venn, onde: M: malhadeira; A: arrastão de praia; T: tarrafa e E: espinhel.

FIGURA 17: Evolução mensal da captura por aparelho de pesca. 
FIGURA 18: Captura (CAPT ( kg )) versus número de lances (LANCES)

FIGURA 19: Captura (CAPT ( $\mathrm{kg}$ )) versus número de pescadores (PESC)

FIGURA 20: Participação relativa (\%) das espécies mais importantes por aparelho, onde: A (arrastão), AM (arrastão-malhadeira), AMT (arrastão-malhadeira-tarrafa), AT (arrastão-tarrafa), EM (espinhel-malhadeira), EMT (espinhel-malhadeira-tarrafa), ET (espinhel-tarrafa), M (malhadeira), MT (malhadeira-tarrafa), T (tarrafa).................

FIGURA 21: Captura ( $\mathrm{t}$ ) mensal de curimatá em relação ao nível $(\mathrm{cm})$ do rio Tocantins.

FIGURA 22: Nível do rio Tocantins (NIVEL (cm)) versus captura de curimatá............

FIGURA 23: CPUE (kg/pescador) da curimatá

FIGURA 24: Captura por unidade de esforço (CPUE) versus captura acumulada (Ki).

FIGURA 25: Logarítmo natural da captura por unidade de esforço (LNCPUE) versus o esforço acumulado (F). 


\section{LISTA DE TABELAS}

TABELA 01: Captura ( $\mathrm{t}$ ) desembarcada no Mercado Municipal de Imperatriz (MA) no ano de 1988.

TABELA 02: Cota média mensal $(\mathrm{cm})$ do rio Tocantins na Estação Itaguatins (TO) no ano de 1988 .

TABELA 03: Captura (kg), Esforço ( ${ }^{\circ}$ de pescadores) e CPUE (kg/pescador) no Mercado Municipal de Imperatriz (MA) no ano de 1988.

TABELA 04: Rendimento total mensal dos pescadores (US\$), Rendimento mensal por pescador (US\$), Rendimento mensal diário por pescador (US\$), Rendimento total mensal dos marreteiros (US\$) e Lucro (\%) por parte dos Marreteiros no Mercado Municipal de Imperatriz (MA) no ano de 1988.

TABELA 05: Variável, coeficiente, erro padrão (E.P.), teste $t(T)$ e probabilidade exata (p) com $\mathrm{n}=12, \mathrm{gl}=1,10$ para a regressão linear entre número de pescadores (PESC) e as capturas mensais (CAPT)

TABELA 06: Relação das espécies comerciais e Captura $(\mathrm{kg})$ no Mercado Municipal de Imperatriz (MA) no ano de 1988

TABELA 07: Índice de diversidade $\left(\mathrm{H}^{\prime}\right)$ e riqueza de espécies desembarcadas no Mercado Municipal de Imperatriz (MA) no ano de 1988.

TABELA 08: Coeficiente de concordância de Kendall (W), estatística de Friedman, graus de liberdade (g.l.) e probabilidade (p), para os postos atribuídos às espécies que ocorreram no desembarque pesqueiro do Mercado Municipal de Imperatriz (MA) durante o ano de 1988

TABELA 09: Captura ( $\mathrm{kg}$ ), Esforço ( $\mathrm{n}^{\mathrm{o}}$ pescadores) e CPUE ( $\mathrm{kg} /$ pescador) no Mercado Municipal de Imperatriz (MA) nos meses de março, abril e novembro de 1988 e 1997.

TABELA 10: Captura $(\mathrm{kg})$ de Siluriformes, Esforço $\left(\mathrm{n}^{\circ}\right.$ pescadores) e CPUE (kg/pescador) no Mercado Municipal de Imperatriz (MA) no mês de dezembro de 1988 e 1997.

TABELA 11: Produção ( $\mathrm{t}$ ) e Participação relativa (\%) dos aparelhos no Mercado Municipal de Imperatriz (MA) no ano de 1988 
TABELA 12: Variável, coeficiente, erro padrão (E.P.), teste (T) e probabilidade exata (p) com $\mathrm{n}=12, \mathrm{gl}=1,10$ para a regressão linear entre as unidades de esforço (UE) e as capturas mensais com malhadeira. $\mathrm{f}_{2}: \mathrm{n}^{\mathrm{o}}$ de pescadores $\mathrm{x}$ dias de pesca e $\mathrm{f}_{3}$ : $\mathrm{n}^{\mathrm{o}}$ de pescadores

TABELA 13: Captura ( $\mathrm{kg}$ ) das espécies mais importantes, grandes bagres e outros pescados por aparelho no Mercado Municipal de Imperatriz (MA) no ano de 1988. A (arrastão), AM (arrastão-malhadeira), AMT (arrastão-malhadeira-tarrafa), AT (arrastão-tarrafa), EM (espinhel-malhadeira), EMT (espinhel-malhadeira-tarrafa), ET (espinhel-tarrafa), M (malhadeira), MT (malhadeira-tarrafa), T (tarrafa).......................

TABELA 14: Estimativa da abundância $(\hat{N})$ do Prochilodus nigricans - curimatá -, intervalo de confiança ao nível de 5\% (limite inferior e superior) e coeficiente de capturabilidade (q) 


\section{RESUMO}

CETRA, M. (1998). Ecologia da pesca artesanal no médio rio Tocantins, Imperatriz (MA).

São Carlos, 1998. 72p. Dissertação (Mestrado) - Escola de Engenharia de São Carlos,

Universidade de São Paulo.

Neste trabalho é analisado um levantamento realizado em 1988 para se quantificar a produção pesqueira na região do médio rio Tocantins, a fim de avaliar a importância da atividade pesqueira para as populações locais. Visa compreender a forma de adaptação das técnicas utilizadas pelos pescadores às características ecológicas das espécies alvo das pescarias, considerando as mudanças ambientais ocorridas durante um ciclo hidrológico. Os peixes adaptaram suas estratégias de vida às variações sazonais, assim como, a comunidade de pescadores se adaptou a esta variabilidade espaço-temporal escolhendo estratégias de captura apropriadas. Os pescadores conseguiram uma seleção das espécies a partir de um conhecimento aprofundado do ambiente, dos organismos que nele habitam e da seletividade dos apetrechos aplicados. Os pescadores ficam obrigados a fornecer toda a sua produção aos primeiros atravessadores em troca do financiamento de gastos na manutenção dos barcos, compra de combustível, manutenção dos apetrechos, e fornecimento de gelo para conservação do pescado. Existe uma preferência em se capturar peixes de escama, como a curimatá Prochilodus nigricans e o jaraqui Semaprochilodus brama, apesar de terem um valor comercial inferior. Esta preferência é determinada pela grande abundância e aceitação no mercado local. Dados recentes de levantamento dos recursos pesqueiros feito pelo Ministério do Meio Ambiente na mesma região, forneceram informações importantes, as quais permitem concluir que alterações nas características físicas e biológicas do médio rio Tocantins, provocadas pela construção da barragem de Tucuruí, propiciaram ao mampará Hipophtalmus marginatus a conquista dos ambientes situados acima desta área. Durante a época de defeso, os Siluriformes passam a ser o alvo das pescarias, o que irá afetar negativamente a estrutura da comunidade de peixes do médio rio Tocantins. Através dos dados de captura por arrastão de praia em 1988, utilizando o número de lances como unidade de esforço, estimou-se a população de curimatá, existente no trecho que vai de Imperatriz (MA) até a zona de confluência entre os rios Araguaia e Tocantins, utilizando-se o método de depleção. Estimou-se uma população em torno de $200 \mathrm{t}$, com um intervalo variando de $174 \mathrm{t}$ a $227 \mathrm{t}$.

Palavras-chave: pesca artesanal; rio Tocantins; depleção 


\begin{abstract}
CETRA, M. (1998). Ecology of the artisanal fishing in the medium river Tocantins, Imperatriz (MA). São Carlos, 1998. 72p. Dissertação (Mestrado) - Escola de Engenharia de São Carlos, Universidade de São Paulo.

In this thesis, a survey carried out in 1988 is analysed in order to quantify the fishing production in the area of the medium river Tocantins, and to evaluate the importance of the fishing activity for the local populations. It is aimed to understand the adaptation of the techniques used by the fishermen to the ecological characteristics of the fisheries target species, considering the environmental changes during a hidrological cycle. The fishes adapted its life strategies to the seasonal variations, as well as, the community of fishermen adapted themselves to this space-temporal variability choosing apropriate capture strategies. The fishermen select the species based in their knowledgement of the environment, and of its organisms through the selectivity of the fishing gears. Fishermen are forced to supply all its production to the first merchant in exchange of financing the expenses in order to maintain the ships, buying fuel, uphold the equipments, and supply ice for fish conservation. A preference exists in capturing scale fish, as the curimatá Prochilodus nigricans and the jaraqui Semaprochilodus brama, in spite of having inferior commercial value. This preference is determined by their abundance and acceptance in the local market. Recent surveys performed by the Ministry of the Environment in the same area, supplied important information, which allow to conclude that alterations in the physical and biological characteristics of the medium river Tocantins, provoked by the construction of the dam of Tucuruí, allowed the mampará Hipophtalmus marginatus the conquest of the environments above this area. During the closed season, Siluriformes becomes the target of the fisheries, which afect will negatively the structure of the fish community. The curimatá population, extending from Imperatriz (MA) till the confluence zone of the rivers Araguaia and Tocantins has been estimated for 1988, using the depletion method, as $200 \mathrm{t}$, with an interval varying from $174 \mathrm{t}$ to $227 \mathrm{t}$.
\end{abstract}

Keywords: artisanal fisheries; river Tocantins; depletion estimates 